\title{
Wing morphing control with shape memory alloy actuators
}

\begin{abstract}
Aircraft morphing is referred to as the ability for an aircraft to change its geometry in flight. Formally, flaps, spoilers, and control devices are considered morphing, but in general, morphing in aerospace is associated with geometrical changes using smart materials such as shape memory alloys. Shape memory alloy is a material that changes shape under heating and produces force and deflections, which make it potential actuator for a wing morphing system. The motivation behind this study is the application to small-sized and medium-sized unmanned air vehicles and the potential to increase range or endurance for a given fuel load through improved lift-to-drag ratio. The camber line of an airfoil section, the predominant parameter affecting lift and drag, is changed by resistive heating of a shape memory alloy actuator and cooling in the surrounding air. Experiments were conducted under wind tunnel conditions to verify analysis and to investigate the effects of its application on the aerodynamic behavior of the wing. This study investigated three control methodologies: the conventional proportionalï integralï derivative controller, proportionalï integralï derivative with robust compensator, and proportionalï integralï derivative with anti-windup compensator. The latter proved to have superior performance in achieving and maintaining a required level of morphing. In addition, the power required to the shape memory alloy actuators under aerodynamic load, and the effect of ambient temperature was also investigated.
\end{abstract}

Keyword: Aerodynamic efficiency; Airfoil camber; Control; Electrostrictive; Morphing; Piezoelectric; Shape memory 